\title{
Retrosternal Hemorrhage after Cardiac Compression
}

\author{
Byung Hee Kang, M.D., Jonghwan Moon, M.D., Kyoungwon Jung, M.D., Yo Huh, M.D.
} Division of Trauma Surgery, Department of Surgery, Ajou University School of Medicine, Suwon, Korea

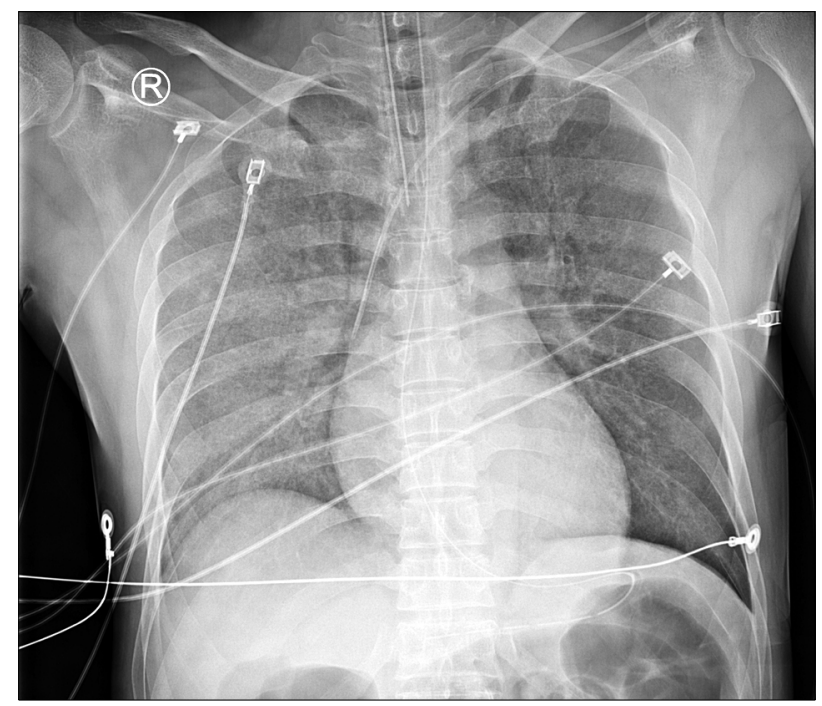

Fig. 1. Chest radiograph showed normal cardiac contour.

A 43-year-old male visited the Trauma Center after a pedestrian traffic accident. During initial resuscitation, suddenly the ventricular fibrillation was detected. Cardiac compression and cardioversion were performed for about 10 minutes, and his rhythm was recovered. A chest radiograph was taken (Fig. 1), and focused assessment with sonography in trauma (FAST) was negative (Pericardial effusion wasn't seen, and heart movement was decreased, However it was difficult to distinguish from the effect of ventricular fibrillation).

He was admitted to the intensive care unit and a transesophageal echocardiography

Received December 14, 2016,

Revised March 13, 2017,

Accepted March 15, 2017

\section{Correspondence to: Yo Huh, M.D.}

Division of Trauma Surgery, Department of Surgery, Ajou University School of Medicine, 164 WorldCup-ro, Yeongtong-gu, Suwon 16499 , Korea

Tel: +82-31-219-7764, Fax: +82-31-219-7781, E-mail: ermdhuhyo@gmail.com

ORCID: http://orcid.org/0000-0002-1220-1534
(TEE) was performed for cardiac evaluation (Unfortunately, the image wasn't saved). TEE presented cardiac tamponade features, hence a large amount of fluid was administrated instead of using more inotropic drug. Computed tomography (CT) scan was taken and the CT showed retrosternal hematoma which compressed the right ventricle (Fig. 2). Because retrosternal hematoma was not easily detected in FAST, CT or TEE could be considered for evaluation after cardiac compression [1,2].

Copyright $@ 2018$ by Korean Society of Acute Care Surgery

(c) This is an Open Access article distributed under the terms of the Creative Commons Attribution Non-Commercial License (http://creativecommons.org/licenses/by-nc/4.0) which permits unrestricted non-commercial use, distribution, and reproduction in any medium, provided the original work is properly cited. 

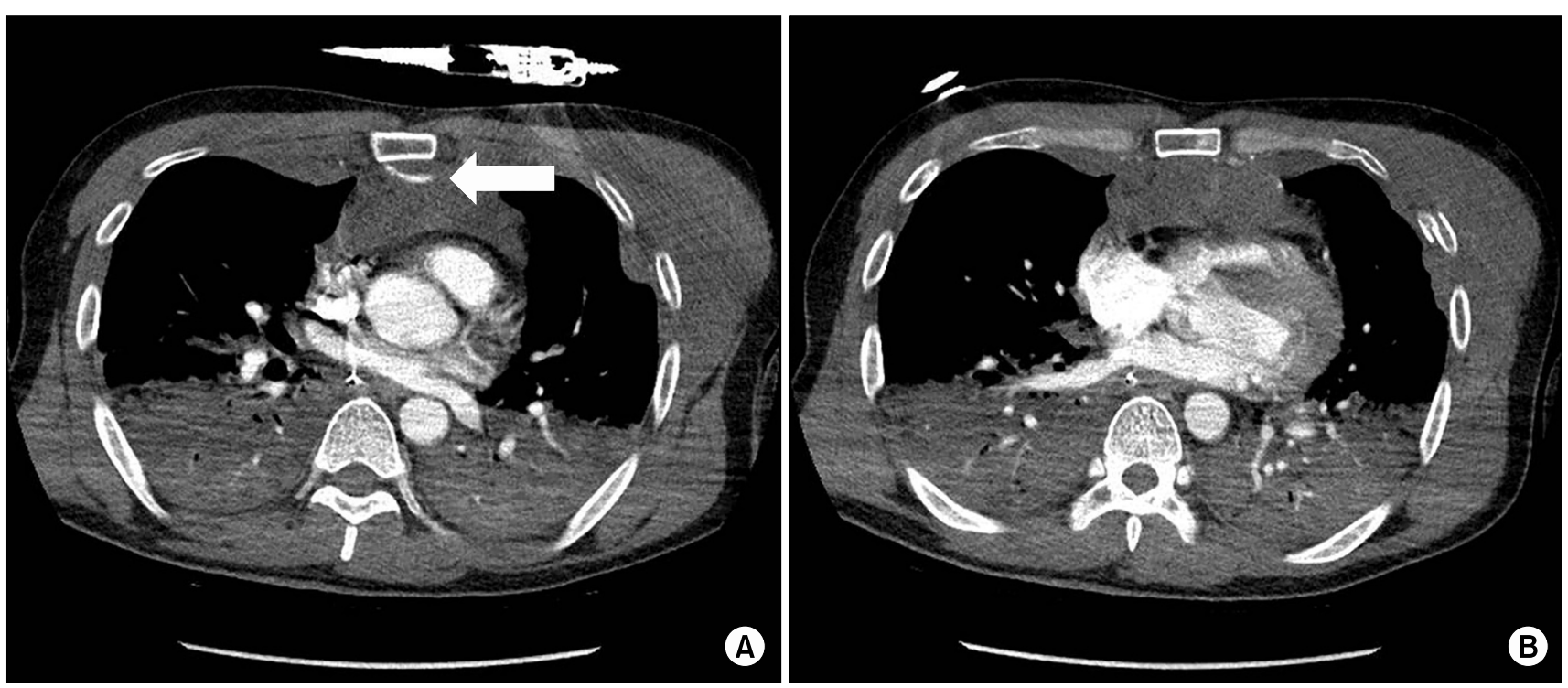

Fig. 2. (A) Indicator showed active bleeding of retrosternal area. (B) Heart was compressed by retrosternal hematoma, and both lung atelectasis was shown.

\section{Conflicts of Interest}

No potential conflict of interest relevant to this article was reported.

\section{References}

1. Hsu LW, Chong CF, Wang TL, Wu BH. Traumatic mediastinal hematoma: a potentially fatal condition that may be overlooked by traditional focused assessment with sonography for trauma. Am J Emerg Med 2013;31:262.e1-3.

2. Kim YH, Kwon JB, Park CB, Choi SY. Extrapericardial cardiac tamponade by a retrosternal haematoma after blunt chest trauma. Eur J Cardiothorac Surg 2012;41:958. 